\title{
Response Surface Space Mapping for Electromagnetic Optimization
}

\author{
Mark Dorica and Dennis D. Giannacopoulos \\ Department of Electrical and Computer Engineering, McGill University, Montreal, QC H3A 2A7, Canada
}

\begin{abstract}
An electromagnetic system can be described in a variety of ways. Coarse models provide fast evaluations but lack the required accuracy in the final stages of design. Fine models are highly accurate, but prohibitively expensive. Finding a compromise between these extremes may assist in overcoming bottlenecks in design automation and optimization. One approach is to carry out optimization in the coarse model space and use fine model simulations to fine-tune the result via space mapping. A new response surface space mapping (RSSM) strategy is presented and applied to an E-shaped patch antenna test case. The solutions that emerge are comparable to full fine model optimization at a fraction of the cost.
\end{abstract}

Index Terms-Electromagnetic modeling, electromagnetic optimization, response surface (RS) models, space mapping (SM).

\section{INTRODUCTION}

W ITHIN the design process, the costs associated with fine models, such as finite elements (FEs), are often prohibitive. Empirical approaches like response surface (RS) models have found success in accurately approximating the FE model [1]-[3]. Further increases in efficiency can be obtained by employing design of experiments (DOE) theory [1]. These methods provide significant speed advantages once the model has been constructed. While building an RS model is a one-time cost, it can nevertheless be a substantial one. A successful RS model of an electromagnetic shaker required 171 magnetostatic solutions [3]. At 15 min per solution, the RS model would be ready in about $40 \mathrm{~h}$.

An alternate strategy, based on space mapping (SM), aims to combine cheap coarse models with highly accurate fine models. A coarse model can be built in the same way as an RS. The coarse model will have inherent errors but is expected to preserve the general character of the device response. An optimization of this coarse model would follow. Fine simulations would then be conducted in an iterative manner and would be used to align the coarse model with the fine model. The fine optimal solution would emerge after a certain number of iterations.

In much of the SM literature (e.g., [4]-[6]), the coarse models are circuit models available within commercial simulators. The behavior of the microwave device over a frequency range is usually the model output that is of interest in existing SM algorithms [6]. SM methods for optimization of a multivariable RS, and multiobjective optimization of several linked RS models have remained absent. In this paper, the response surface space mapping (RSSM) technique is introduced for the first time. As will be seen, RSSM is also directly applicable to low-frequency optimization problems in electromagnetics.

\section{RESPONSE SuRface SPACE MAPPING}

The behavior of an electromagnetic device is described in both the coarse model space $X_{c}$ and the fine model space $X_{f}$.

Digital Object Identifier 10.1109/TMAG.2006.872018

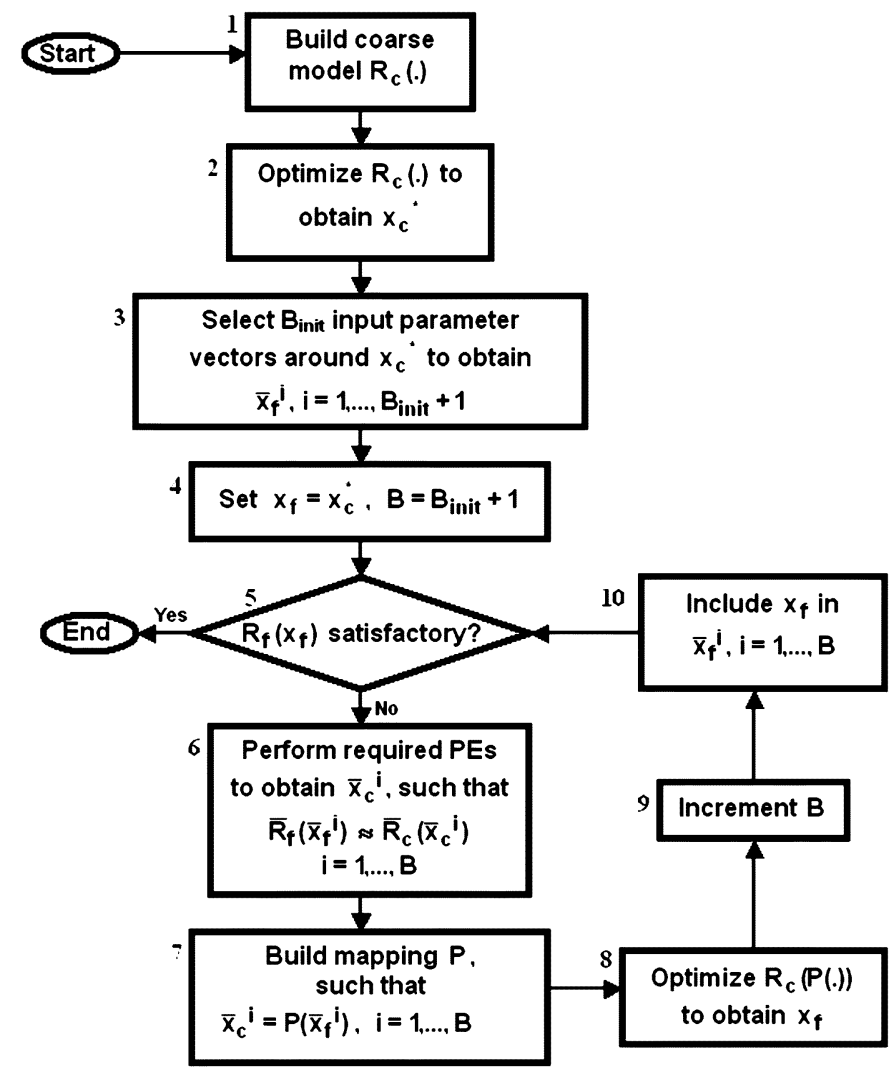

Fig. 1. Response surface space mapping (RSSM).

Design parameters are represented by vectors $x_{c}$ and $x_{f}$ in $X_{c}$ and $X_{f}$, respectively. Coarse and fine model responses are denoted by $R_{c}(\cdot)$ and $R_{f}(\cdot)$, respectively. The RSSM flowchart for a RS coarse model is given in Fig. 1.

In step 3 of Fig. 1, the $B_{\text {init }}$ input parameters are selected to provide a starting pool of data for the RSSM algorithm (total of $B_{\text {init }}+1$ points when $x_{c}^{*}$ is included). The reason for selecting these points near $x_{c}^{*}$ is that the true optimum is expected to be in the vicinity. The key RSSM operations occur within steps 5-10 of the loop. At each iteration, the parameter extraction (PE) phase of step 6, implemented via genetic algorithm (GA) [7], finds the inputs to the coarse model that will yield a 
response as similar as possible to the fine response of step 5. The $\mathrm{PE}$ result is then added to the set $\bar{x}_{c}^{i}$. It is important to note that in the first iteration of the loop, $B_{\text {init }}+1$ PE's are performed in step 6. In subsequent iterations, a single PE is performed based on the newly computed $R_{f}\left(x_{f}\right)$ of step 5 . Let the $B_{\text {init }}$ input parameters selected in step 3 be denoted by $x_{\text {init }}^{i}, i=1, \ldots, B_{\text {init }}$. The $m$ th PE operation takes the form

$$
\bar{x}_{c}^{m}=\arg \min _{x_{c}}\left\|\bar{R}_{c}\left(x_{c}\right)-\bar{R}_{f}\left(\bar{x}_{f}^{m}\right)\right\|
$$

with

$$
\begin{aligned}
\bar{R}_{f}\left(\bar{x}_{f}^{m}\right) & =\left[R_{f}\left(\bar{x}_{f}^{m}\right), R_{f}\left(x_{\text {init }}^{1}\right), R_{f}\left(x_{\text {init }}^{2}\right), \ldots, R_{f}\left(x_{\text {init }}^{B_{\text {init }}}\right)\right] \\
\bar{R}_{c}\left(x_{c}\right) & =\left[R_{c}\left(x_{c}\right), R_{c}\left(x_{\text {aug }}^{1}\right), R_{c}\left(x_{\text {aug }}^{2}\right), \ldots, R_{c}\left(x_{\text {aug }}^{B_{\text {init }}}\right)\right] \\
x_{\text {aug }}^{i} & =x_{c}+x_{\text {init }}^{i}-\bar{x}_{f}^{m}, \quad i=1, \ldots B_{\text {init }} .
\end{aligned}
$$

The aim of (2)-(4) is to augment the responses $R_{c}\left(x_{c}\right)$ and $R_{f}\left(\bar{x}_{f}^{m}\right)$ that have a single value as output. Using $R_{c}(\cdot)$ and $R_{f}(\cdot)$ alone would not be meaningful because a single output value is not sufficient to characterize the behavior of the model; the result of the GA search would surely not be unique. Each additional computation of (1) only entails a single fine model evaluation (in step 5 of Fig. 1) because the evaluations at $x_{\text {init }}^{i}, i=$ $1, \ldots B_{\text {init }}$ are continually reused. This PE strategy allows for similarity to be evaluated "within" the RS. Previous strategies used fine model evaluations over a frequency range in order to evaluate similarity and were not applicable to general multivariable, single-output RS models.

Following PE, a mapping $P$ is built in step 7 using all previously computed fine responses and results of PE. The role of this mapping is to transform the fine input parameters so that $R_{c}(P(\cdot))$ in step 8 behaves like the fine model. The mapping is implemented using an artificial neural network (ANN) [8]. The RSSM algorithm essentially builds and enhances the mapped coarse model until a desirable optimum is achieved. Each iteration of RSSM provides additional information for building the mapping.

RSSM can be directly generalized to multiple coarse models that are linked for solving an optimization problem. For instance, power, efficiency, and size could each be represented as a separate RS and jointly optimized. Thus, the overall coarse model would be comprised of $K$ multivariable, single-output RS models. All RSSM steps would be performed in an analogous fashion, with separate $\mathrm{PE}$ and $\mathrm{ANN}$ mappings required for each submodel. For this general case of $K$ submodels, (5)-(9) give a synopsis of the costs associated with the RSSM technique. For simplification of the expressions, we are assuming that the number of iterations is greater than zero. For the trivial case of no iterations, it is readily apparent that only a single coarse model optimization and single fine model evaluation would be required, signifying that the optimum was achieved at the first attempt. The costs associated with the proposed technique fall roughly into five categories: fine model evaluations (5); PE (6); enhanced model optimization (7); ANN model building (8); and coarse model building and optimization (9). In the expressions that follow, fine ${ }_{c}, \mathrm{pe}_{c}, \mathrm{ann}_{c}, \mathrm{samp}_{c}$, and $\mathrm{crs}_{c}$ represent per action costs for fine evaluations, PE, ANN building, coarse evaluations for RS model building, and enhanced/coarse model optimization, respectively. The variables iters and samps are the number of RSSM iterations and total coarse samples for RS building (per RS model), respectively

$$
\begin{aligned}
\mathrm{FINE}_{c} & =\text { fine }_{c} \times K \times\left(B_{\text {init }}+\text { iters }+1\right) \\
\mathrm{PE}_{c} & =\mathrm{pe}_{c} \times K \times\left(B_{\text {init }}+\text { iters }\right) \\
\mathrm{ENH}_{c} & =\mathrm{crs}_{c} \times \text { iters } \\
\mathrm{ANN}_{c} & =\operatorname{ann}_{c} \times K \times \text { iters } \\
\mathrm{CRS}_{c} & =\operatorname{samp}_{c} \times K \times \text { samps }+\mathrm{crs}_{c} .
\end{aligned}
$$

In much of the previous literature (e.g., [6]), the termination criteria were tied to achieving a fine response that is equal to the (desirable) optimal coarse response. In the current paper, the coarse models may be arbitrarily poor. The objective of these coarse models is to generally approximate the character of the device response. They are inherently insufficient for standalone optimization. Thus, the termination criteria relate to the overall quality of the optimum rather than its proximity to the coarse optimum. Novel features of the RSSM technique include the following:

1) submodel (RS) based;

2) use of all available fine data for development of mapping;

3) independent PE and ANN for each submodel, allowing independent evolution;

4) no additional fine model evaluations required for determining similarity in the RSSM loop; data is reused, and each fine simulation is a potential fine optimum.

\section{E-ShAPED PATCH ANTENNA}

The E-shaped patch antenna test case is shown in Fig. 2.

The E-shaped patch antenna is a well-known configuration [9] and is also the subject of a patent [10]. It will be used to provide insight into the performance of RSSM. Let us assume a starting design (in millimeters)

$$
\begin{aligned}
(L, W, h) & =(70,45,10),\left(X_{f}, Y_{f}\right)=(35,7) \\
L_{s} & =35 \quad W_{s}=4 \quad P_{s}=9 .
\end{aligned}
$$

The width $W$ of the antenna controls the higher resonant frequency, whereas the slots $\left(L_{s}, W_{s}, P_{s}\right)$ control the lower resonant frequency. Let us state the design objective of minimizing the maximum $\left|\mathrm{S}_{11}\right|$ at 2.21 and $2.58 \mathrm{GHz}$. The coarse models were built based on a uniform grid of 100 data points. The first design variable $W$ is shown as a vertical dashed line in Fig. 2. The width $W$ is sampled at ten equally spaced points between 36 and $62 \mathrm{~mm}$ (along the $y$ axis). The second design variable is shown by the two horizontal dashed lines and denoted by $H$ and $H^{\prime}$. The $H$ parameter, whose starting design value is $46 \mathrm{~mm}$, is varied through ten sample points between 43 and $69 \mathrm{~mm}$ (along the $x$ axis). The lower horizontal line $H^{\prime}$ moves opposite to $H$ (when $H$ moves up, $H^{\prime}$ moves down and so on).

\section{RESULTS}

The antenna $\left|\mathrm{S}_{11}\right|$ at 2.21 and $2.58 \mathrm{GHz}$, as a function of $W$ and $H$, leads to two separate two-variable submodels. The 


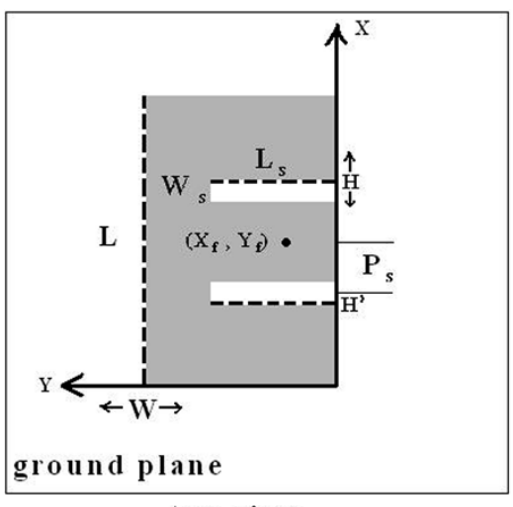

top view

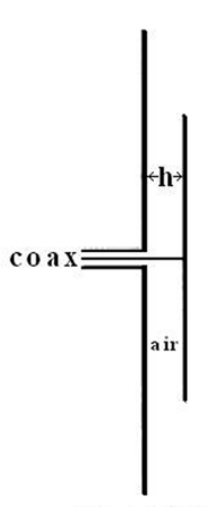

side view
Fig. 2. Geometry of E-shaped patch antenna test case.

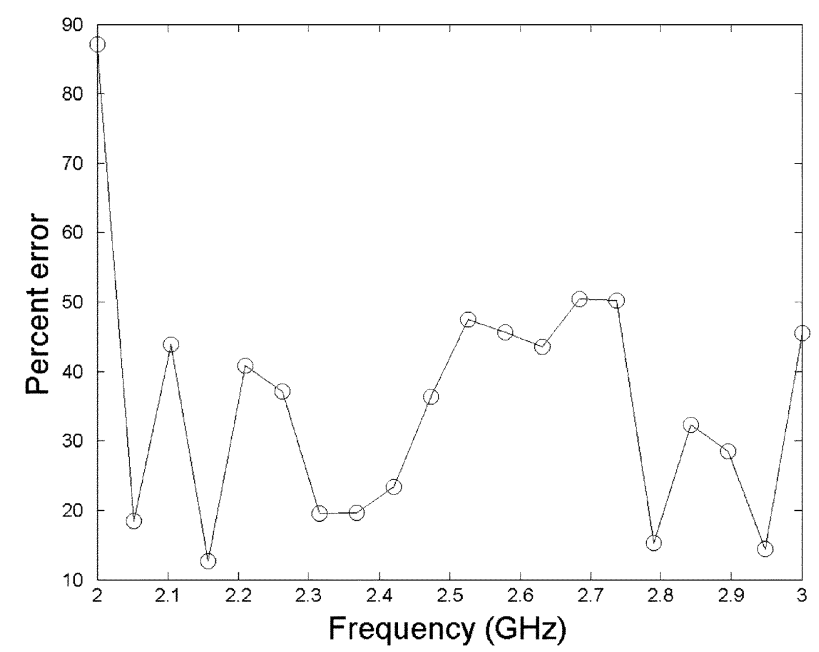

Fig. 3. Error in E-shaped antenna coarse model.

coarse data required for these submodels is $100 \times 2=200$ evaluations. Let us begin by investigating the resulting coarse model. Fig. 3 shows the behavior of the coarse model average percent error over a wide range of frequencies. At the two frequencies of interest, the accuracy is particularly poor, ranging between $40 \%$ and $50 \%$ error. For most purposes, this model would be unusable. However, here it is used as a challenging test case for RSSM.

The overall accuracy performance is depicted in Fig. 4, with $x_{c}^{*}, x_{\mathrm{sm}}^{*}$, and $x_{f}^{*}$ denoting the coarse, SM, and fine optimal solutions, respectively. The optimization approaches were applied to the two frequency points of interest, but the full fine model response from 2 to $3 \mathrm{GHz}$ is given for completeness. The coarse and fine optimizations were carried out using a standard GA [7]. The coarse model optimal design $x_{c}^{*}$, as evaluated within the coarse model $R_{c}\left(x_{c}^{*}\right)$, was actually the best design of all (not shown). In Fig. 4, however, it is observed that $x_{c}^{*}$ does not produce an adequate fine model performance $R_{f}\left(x_{c}^{*}\right)$. This initial unsatisfactory design is the starting point for RSSM. Note in Fig. 4 that RSSM produces a greatly improved design $x_{\mathrm{sm}}^{*}$ which has a response $R_{f}\left(x_{\mathrm{sm}}^{*}\right)$ that is significantly different from the optimal coarse result. The GA applied to the fine model produces a design $x_{f}^{*}$ with a virtually identical response to the RSSM result. Convergence of the GA optimization of the fine

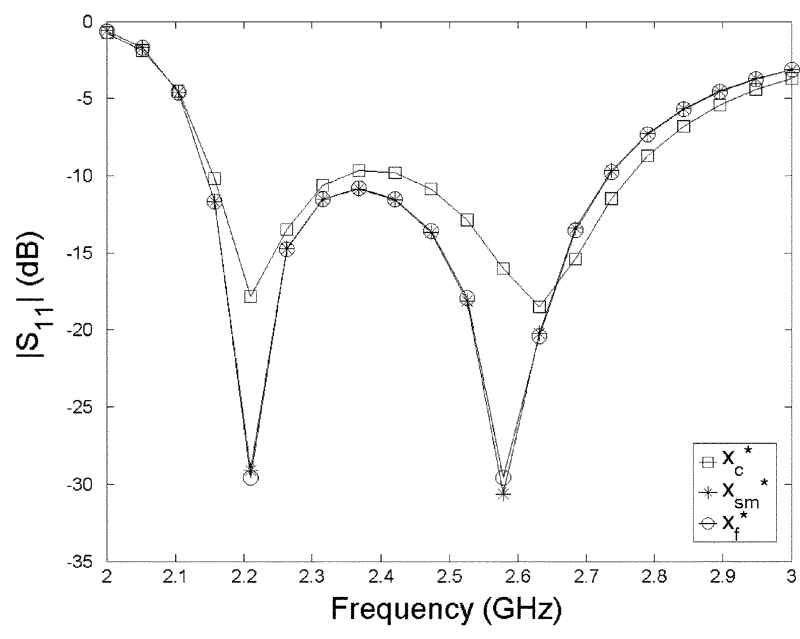

Fig. 4. Antenna responses resulting from various optimization methods.

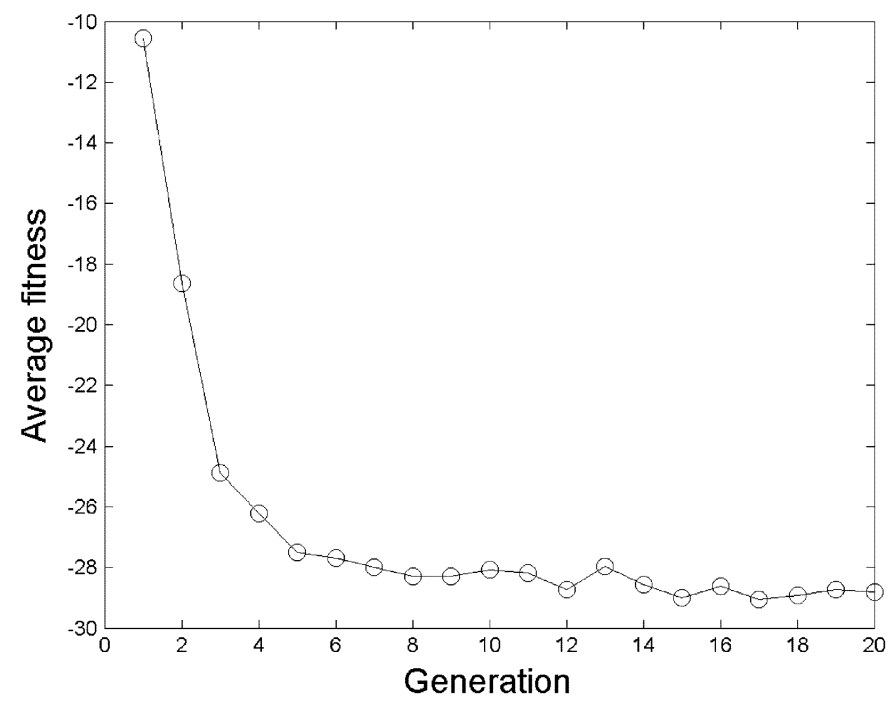

Fig. 5. Convergence of genetic algorithm optimization of fine model.

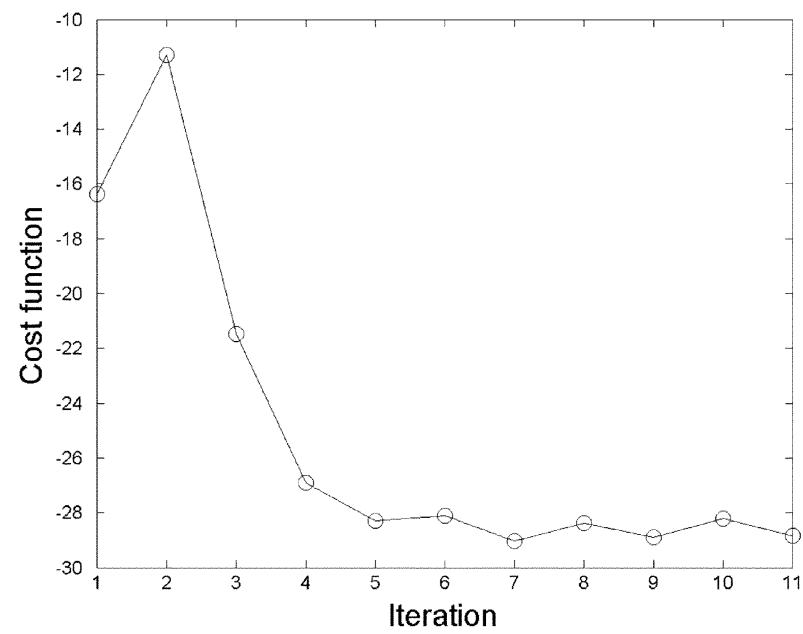

Fig. 6. Convergence of RSSM.

model is shown in Fig. 5. The convergence behavior of RSSM is shown in Fig. 6. The cost function represents the maximum $\left|\mathrm{S}_{11}\right|$ over the two frequency points. The optimal design was actually attained after only seven iterations of RSSM. 
TABLE I

DESIGNS FROM OPTIMIZATION TECHNIQUES

\begin{tabular}{cccc}
\hline Parameter & Coarse $\left(x_{c}{ }^{*}\right)$ & Fine $\left(x_{f}^{*}\right)$ & $\operatorname{RSSM}\left(x_{s m}{ }^{*}\right)$ \\
\hline$W(\mathrm{~mm})$ & 47.22 & 48.59 & 48.65 \\
\hline$H(\mathrm{~mm})$ & 47.17 & 45.78 & 45.72 \\
\hline
\end{tabular}

TABLE II

SUMMARY OF OPTIMIZATION COSTS

\begin{tabular}{cccc}
\hline Optimization & Action & Time (s) & \% of total \\
\hline \multirow{3}{*}{ Coarse (GA) } & Model building & 199.19 & 97.0 \\
\cline { 2 - 4 } & Optimization & 6.16 & 3.0 \\
\cline { 2 - 4 } & Total & 205.35 & 100 \\
\hline \multirow{3}{*}{ RSSM } & Fine evaluations & 90.47 & 11.5 \\
\cline { 2 - 4 } & PE & 367.71 & 46.8 \\
\cline { 2 - 4 } & Enhanced opt. & 93.30 & 11.9 \\
\cline { 2 - 4 } & ANN building & 32.51 & 4.2 \\
\cline { 2 - 4 } & Coarse costs & 201.13 & 25.6 \\
\cline { 2 - 4 } & Total & 785.12 & 100 \\
\hline \multirow{3}{*}{ Fine (GA) } & Fine evaluations & 3209.90 & 0.9 \\
\cline { 2 - 4 } & GA operations & 28.50 & 100 \\
\cline { 2 - 4 } & Total & 3238.40 & \\
\hline
\end{tabular}

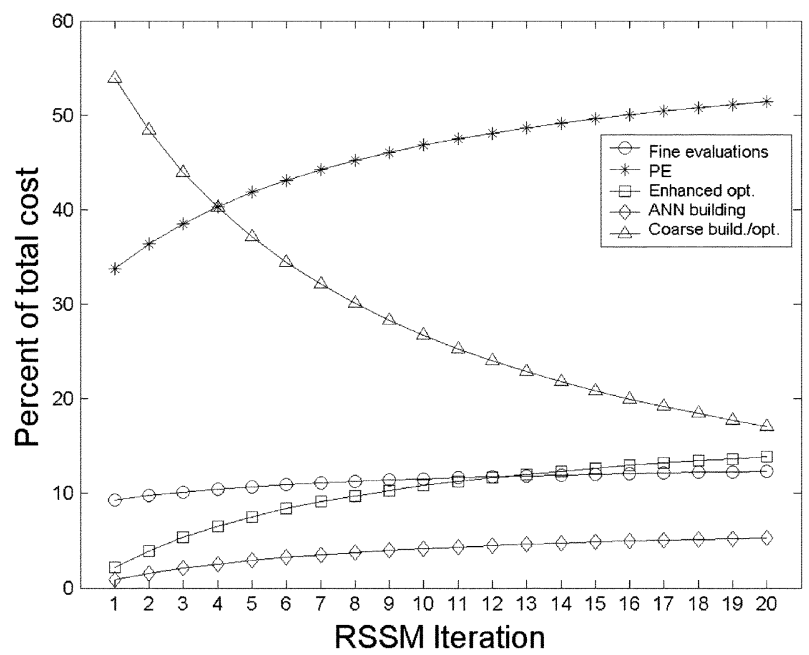

Fig. 7. Evolution of RSSM costs.

Table I shows the final designs from the optimization approaches. Notice the evolution of the RSSM technique starting with the coarse optimum and arriving at the RSSM optimum.

Numerical results associated with the costs of the optimization techniques are presented in Table II. While RSSM is roughly four times more costly than coarse optimization for this test case, it only incurs $25 \%$ of the time cost of a standard GA optimization of the fine model. The GA requires 1078 fine evaluations, and RSSM only 30.

The behavior of the costs with increasing iteration number is shown in Fig. 7. We note that PE, which is the key step in the RSSM technique, is also the main contributor to the overall cost of the technique.

\section{CONCLUSION}

A response surface space mapping (RSSM) technique has been proposed for electromagnetic optimization. RSSM begins with multiple coarse response surface models built using simulation data. The RSSM algorithm takes advantage of the implicit knowledge in these coarse models to iteratively construct and optimize an enhanced model based on mappings between input parameter spaces. The enhanced response surface (RS) models are allowed to evolve independently. The bulk of the costs are transferred from fine model evaluations to mapping operations between the input spaces. The RSSM technique employs artificial neural network (ANN) and genetic algorithm (GA) in a supporting role, but these could be replaced by analogous methods. An E-shaped antenna test case was used for demonstration. RSSM was able to achieve solutions that are comparable to full fine model optimization at a fraction of the cost. RSSM can similarly be applied to low-frequency electromagnetic optimization.

Cases inevitably arise where a space mapping is insufficient to transform the coarse model into a good approximation of the fine model. In such cases, output mappings have been suggested [6]. Future research could focus on incorporating output mappings in the RSSM framework.

\section{ACKNOWLEDGMENT}

This work was supported in part by the Natural Sciences and Engineering Research Council of Canada and Le Fonds québécois de la recherche sur la nature et les technologies.

\section{REFERENCES}

[1] D. A. Lowther, "Automating the design of low frequency electromagnetic devices-A sensitive issue," Int. J. Comput. Math. Elec. Electron. Eng., vol. 22, no. 3, pp. 630-642, 2003.

[2] Z. Malik and K. Rashid, "Comparison of optimization by response surface methodology with neurofuzzy methods," IEEE Trans. Magn., vol. 36, no. 1, pp. 241-257, Jan. 2000.

[3] D. N. Dyck and B. S. Murray, "Transient analysis of an electromagnetic shaker using circuit simulation with response surface models," IEEE Trans. Magn., vol. 37, no. 5, pp. 3698-3701, Sep. 2001.

[4] J. W. Bandler et al., "Space mapping technique for electromagnetic optimization," IEEE Trans. Microw. Theory Tech., vol. 42, no. 12, pp. 2536-2544, Dec. 1994.

[5] J. W. Bandler et al., "Neuromodeling of microwave circuits exploiting space mapping technology," IEEE Trans. Microw. Theory Tech., vol. 47, no. 12 , pp. 2417-2427, Dec. 1999.

[6] J. W. Bandler et al., "Space mapping: The state of the art," IEEE Trans. Microw. Theory Tech., vol. 52, no. 1, pp. 337-361, Jan. 2004.

[7] G. F. Uler, O. A. Mohammed, and C.-S. Koh, "Utilizing genetic algorithms for the optimal design of electromagnetic devices," IEEE Trans. Magn., vol. 30, no. 6, pp. 4296-4298, Nov. 1994.

[8] P. Burrascano, S. Fiori, and M. Mongiardo, "Review of artificial neural networks applications in microwave computer-aided design," Int. J. RF Microw. CAE, vol. 9, no. 3, pp. 158-174, 1999.

[9] F. Y. Yang, X.-X. Zhang, X. Ye, and Y. Rahmat-Samii, "Wide-band E-shaped patch antennas for wireless communications," IEEE Trans. Antennas Propag., vol. 49, no. 7, pp. 1094-1100, Jul. 2001.

[10] M. Sanad, "Small double C-patch antenna contained in a standard PC card," U.S. Patent 5657 028, Aug. 12, 1997.

Manuscript received June 20, 2005 (e-mail: dennis.giannacopoulos @ mcgill.ca). 